\title{
Synthesis of Non-Uniform Amplitude equally Spaced Antenna Arrays Using PSO and DE Algorithms
}

\author{
K. Yasoda ${ }^{1}$, K.Karuna Kumari ${ }^{2}$ \\ ${ }^{1}$ Research Scholar, Department of ECE, GITAM University, Visakhapatnam, A.P., INDIA \\ ${ }^{2}$ Associate Professor, Department of ECE, GITAM University, Visakhapatnam, A.P., INDIA
}

\begin{abstract}
In recent years Evolutionary computation has its growth to extent. Synthesis of non-uniform linear antenna arrays is one of the most important electromagnetic optimization problems of the current interest. In this paper, the design of non-uniform linear antenna arrays with optimum side lobe level reduction is investigated. Two global evolutionary optimization methods are Particle Swarm Optimization algorithm(PSO) and Differential Evolution algorithm(DE) are used to determine an optimum set of weights and positions that provide a radiation pattern with optimum side lobe level having uniform progressive phase between the elements. Simulation results show considerable enhancements in array performances using the global optimizers. The paper finally illustrates a comparative evaluation of the two proposed algorithms regarding their applicability as numerical optimization techniques.
\end{abstract}

Keywords: Linear Antenna arrays, Side lobe level (SLL), Differential Evolution (DE), Particle Swarm Optimization algorithm (PSO).

\section{Introduction}

Low side lobe antennas are becoming an increasingly important component of high performance electronic systems, particularly those operating in heavy clutter and jamming environments. Practically, the radiation pattern of antenna array has not only having the main beam but also side lobes .Most of the power is confined in main beam which provides the coverage in desired portion. Some of the power is also remained in sidelobe levels that are nothing but wastage of transmitting power [19]. If the sidelobe levels are very high, large amount of transmitting power is wastage. For efficient use of transmitting power and desired radiation characteristics can be achieved by using this five following control methods: 1) The geometrical configuration of the overall array 2) The relative displacement between the elements 3) The excitation amplitude of the individual elements 4) The excitation phase of individual elements 5) The relative pattern of the individual elements .Various analytical and numerical methods have been used to optimize the side lobe levels relative to the main beam by using above controls.

Several new optimization techniques have been emerged in the past two decades, that mimic biological evolution, or the way biological entities communicate in nature. Some of these algorithms have been used successively in many electromagnetic and antenna problems include Genetic Algorithms (GA) [1], Particle Swarm Optimization (PSO) [2], and the method of Differential Evolution (DE) Algorithm [3]. In the electromagnetism research area, mainly the antenna synthesis, the use of these optimizers is widely and clearly appreciated. To date, different GA, PSO and DE algorithms have been successfully applied to different problems including antenna design and the array synthesis.

Many efforts have also been devoted to compare these algorithms to each other. Typically, such comparisons have been based on artificial numerical benchmark problems. The goal of many studies was to verify that one algorithm outperformed another on a given set of problems. In general, it has been possible to improve a given standard method within a restricted set of benchmark problems by making minor modifications to it.

Recently, particle swarm optimization (PSO) and differential evolution (DE) have been introduced and particularly PSO has received increased interest from the EC community. Both techniques have shown great promise in several real-world applications [4]-[7]. In this paper, we investigated the performance of DE, PSO for the optimization of SLL.

In the following part of the paper ,the theory and formulation of the optimization problem will be explained.Afterwords, the details of the proposed ensemble based Particle Swarm Optimization (PSO) and Differential Evolution (DE) algorithms will be given. Finally, the application of algorithms in the optimization of SLL will be demonstrated for 32 element antenna array. The corresponding results of two algorithms will be compared. 


\section{Design Of Linear Array Antennas}

Consider a Linear array of $2 \mathrm{~N}$ symmetric array elements uniformly spaced of distance $\mathrm{d}$ between the elements in $\mathrm{x}-\mathrm{z}$ plane. The array factor for the linear antenna array is shown below[8].

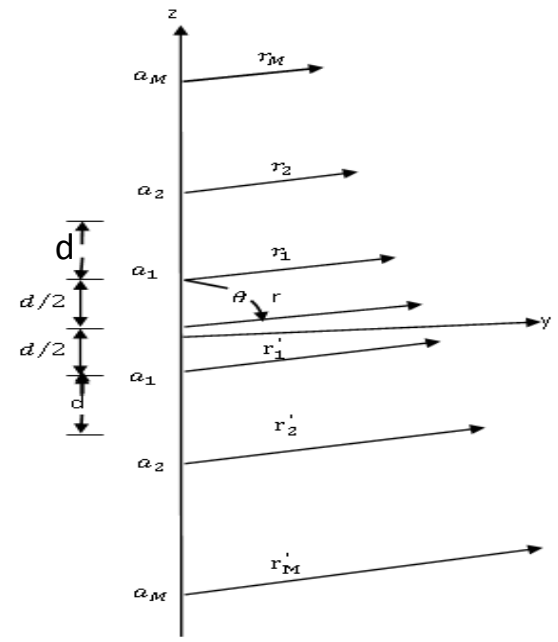

Fig 1: Geometry of Linear Antenna Array

$$
A F(\theta)=2 \sum_{n=0}^{N} I_{N} e^{j \Phi n} \cos [k(n-0.5) d \cos (\theta-\cos \theta 0)]
$$

Where $\mathrm{k}$ is the wave number, $\mathrm{k}=2 \Pi / \lambda, \theta$ is the angle measured from the axis of the array, $\theta \mathrm{o}$ is the steering angle, $\left[I_{N}, \Phi n\right]$ is the excitation of the current for the $n^{\text {th }}$ element on either side of the array midpoint to be controlled and $\mathrm{d}$ is the spacing between elements.By the array factor, the fitness function is obtained.Here, the fitness function is used to obtain the optimum weights and to achieve maximum SLL.

\section{Practical Swarm Optimization Algorithm}

Practical swarm optimization (PSO) is a population-based self-adaptive search optimization technique first introduced by Kennedy and Eberhart [9]. The PSO method is becoming very popular due to its simplicity of implementation and ability to quickly converge to a reasonably good solution. In the practical swarm algorithm, the trajectory of each individual in the search space is adjusted by dynamically altering the velocity of each particle, according to its own flying particles in the D-dimensional search space can be represented as $X_{i}=$ $\left(x_{i 1}, x_{i 2}, \ldots \ldots x_{i D}\right)$ and $V_{i}=\left(v_{i 1}, v_{i 2}, \ldots \ldots v_{i D}\right)$ respectively.

To update the velocity matrix at each iteration $\mathrm{K}$, every particle should now its personal best and global best position vectors in addition to the neighbor best position. The personal best position vector defines the position at which each particle attained its best fitness value up to the present iteration. The personal best position of the $i^{t h}$ particle is represented as $P_{\text {best } 1}=\left(P_{\text {best } i 1}, P_{\text {best } i 2}, \ldots \ldots P_{\text {best iD }}\right)$. the global best position vector defines the position in the solution space at which the best fitness value was achieved by all particles, and is defined by $G_{\text {best } 1}=\left(g_{\text {best } i 1}, g_{\text {best i2 }}, \ldots \ldots g_{\text {best iD }}\right)$.

The particles are manipulated according to the following equations:

$$
\begin{aligned}
v_{i d}^{k+1}= & w v_{i d}^{k}+C_{1} \operatorname{rand}_{1}\left(P_{\text {best }_{i d}}-x_{i d}^{k}\right)+C_{2} \operatorname{rand}_{2}\left(g_{\text {best }_{i d}}-x_{i d}^{k}\right) \\
& x_{i d}^{k+1}=x_{i d}^{k}+v_{i d}^{k+1} \Delta t
\end{aligned}
$$




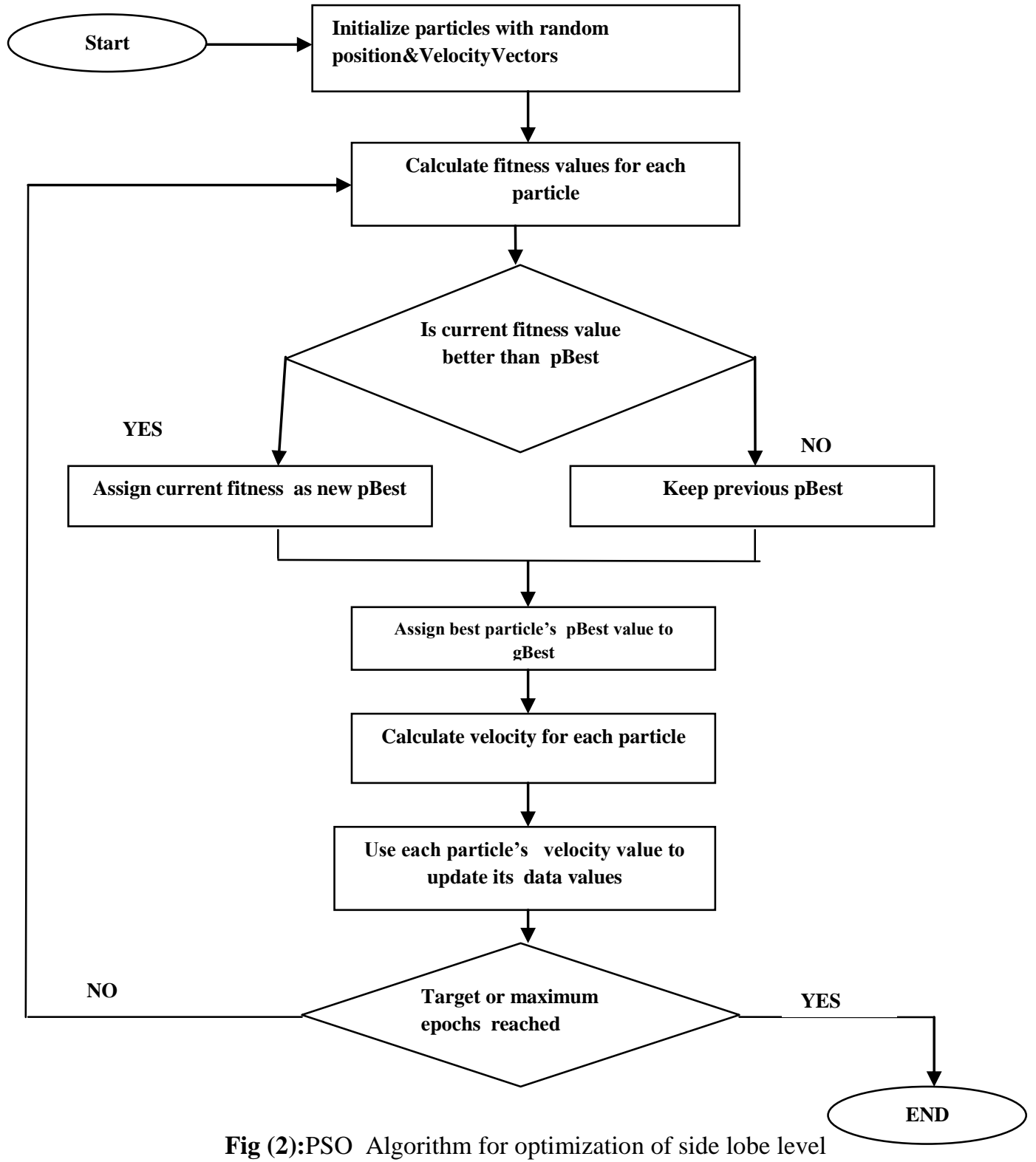

Where $C_{1}, C_{2}$ are the acceleration constants, which represent the weighting of stochastic acceleration terms that pull each particle towards $P_{\text {best }}, g_{\text {best }}$ positions. rand $_{1}$ and rand $_{2}$ are too random numbers in the range of $[0,1], \mathrm{W}$ is the inertia weight introduced to balance between the global and local search abilities. It is set according to the following equation

$$
\mathbf{W}=\boldsymbol{W}_{\max }-\frac{W_{\max }-W_{\min }}{T} . \tau
$$

Where $W_{\max }$ and $W_{\min }$ maximum and minimum $(0.4,0.9)$ is value of the weighting factor respectively. $\mathrm{T}$ is the maximum number of iterations and $\tau$ is the current iteration number. The whole process of PSO algorithm is represented as flowchart as shown in $\operatorname{Fig}(3)$.

\section{De Algorithm}

In 1995, Price and Storn commenced the Differential Evolution (DE) algorithm [10]which is based upon differential mutation operator. Practically there are many problems with different types of objective functions such as non-linear, noisy, flat, non-differentiable, non-continuous, and multi-dimensional or have many local minima which are difficult to solve analytically. DE is a robust statistical method for cost function minimization which does not make use of a single nominal parameter vector but instead it uses a population of equally important vectors and is very advantageous to find the most approximate solution to any type of problems. 
Differential evolution is a simple, efficient and robust evolutionary algorithm, and is usually mark as $\mathrm{DE} / \mathrm{x} / \mathrm{y} / \mathrm{z}$, where $\mathrm{x}$ denotes how the differential mutation base is chosen, $\mathrm{y}$ denotes the number of vectors differences added to the base vector and $\mathrm{z}$ indicates the crossover method.It has been successfully applied to the array synthesis problems, electromagnetic inverse problems and many other scientific and engineering problems. Although it has been reported that differential evolution performs better than many other algorithms, it is still a dream for differential evolution users to have a strategy perfectly balancing exploration and exploitation, or equivalently, reliability and efficiency

It has been well known that the critical idea behind the success of Differential Evolution is the creative invention of differential mutation[11]. Different differential mutation strategies balance exploration and exploitation differently. For example, DE/Best/*/* generally converges faster due to the guidance by the best individual but may be locally trapped because of loss of directivity, while DE/Rand/*/* gains directivity at the cost of efficiency.In order to simultaneously, provide both diversity and guidance so that exploration and exploitation can be more efficiently balanced. Synthesis capability, reliability and efficiency of DE/Rand/*/* are tested. The simulation results show that DE/Rand/*/* is able to achieve lower peak side lobe levels and converge reliably and efficiently.

In $\mathrm{DE}$ algorithm the objective function is sampled by a set of initial points which are chosen randomly from the entire search space. Then in the next step the algorithm adds the weighted difference between the two randomly selected population vectors to the third random population vector to generate a new parameter vector. This process of generating the new parameter vector is called mutation. Now this parameter vector is further mixed with the predefined parameters to produce the trial vector and this process is called crossover. Then in the last step, called selection in which trial vector is replaced by the target vector if the trial vector reduces the values of the cost function then that obtained due to the target vector.

To realize the algorithm let the problem is function of D number of independent parameters. In this work for $\mathrm{N}$ element array, the number of independent parameters is $2 \mathrm{~N}$ in which the first $\mathrm{N}$ parameters are the normalized amplitude coefficient of the $\mathrm{N}$ array elements and remaining $\mathrm{N}$ parameters are to represent the static phase of each element. Hence, if NP be the population size then the parameter vectors are represented as

$$
X_{k, G}=\left[X_{1, i, G}, X_{2, k, G}, \ldots . X_{D, k, G}\right] \mathbf{i}
$$

Where $i=1,2,3 \ldots N P$ and $G$ is the generation number.

The four basic steps of DE algorithm are as follows:

\section{Step-1 Initialization:}

If the upper and lower bounds for each parameter are defined as $x_{i}^{l} \leq X_{2, k, 1} \leq x_{i}^{u}$ for $\mathrm{i}=1,2 \ldots \ldots \mathrm{D}$, then the NP number of D dimensional vectors are randomly generated from the interval $\left[x_{i}^{l}, x_{i}^{u}\right]$ to initiate the searching process.

\section{Step-2 Mutation:}

In this stop the search space is expanded from its initial locations. Two vectors $X_{r 1, G}$ and $X_{r 2, G}$ are selected randomly for a $k^{\text {th }}$ target vector $X_{k, G}$. Let $X_{b e s t, G}$ is the best vector if the current population then the donar vector $V_{i, G}$ is formed as in (6)

$$
V_{i, G}=X_{b e s t, G}+F\left(X_{r 1, G}-X_{r 2, G}\right)
$$

Where $\mathrm{F}$ is the mutation factor and the range of $\mathrm{F}$ is $(0,2)$.

\section{Step-3 Crossover:}

In this step the solutions from the previous generations is obtained successfully, trial vector $V_{k, G}$ is obtained by exchanging the elements of the target vector $X_{K, G}$ and the donar vector $V_{k, G}$ with a cross probability $\mathrm{CR}[0,1]$.

\section{Step-4 Selection:}

The best value for the next generation is selected as

$$
X_{K, G+1}=\left\{\begin{array}{l}
U_{i, G}, \text { if } \mathbf{f}\left(U_{i, G}\right) \leq \mathbf{f}\left(X_{i, G}\right) \\
X_{i, G}, \text { if } \mathbf{f}\left(U_{i, G}\right)>f\left(X_{i, G}\right)
\end{array}\right\}
$$


For $\mathrm{i}=1,2,3 \ldots . \mathrm{NP}$.

The target vector compared with the trial vector and the minimum value is admitted to the next generation. The above steps are continued until the predefined number of generations reached or the desired value of the cost function is obtained.The entire process of DE for solving problem is shown in flowchart in Fig 4.

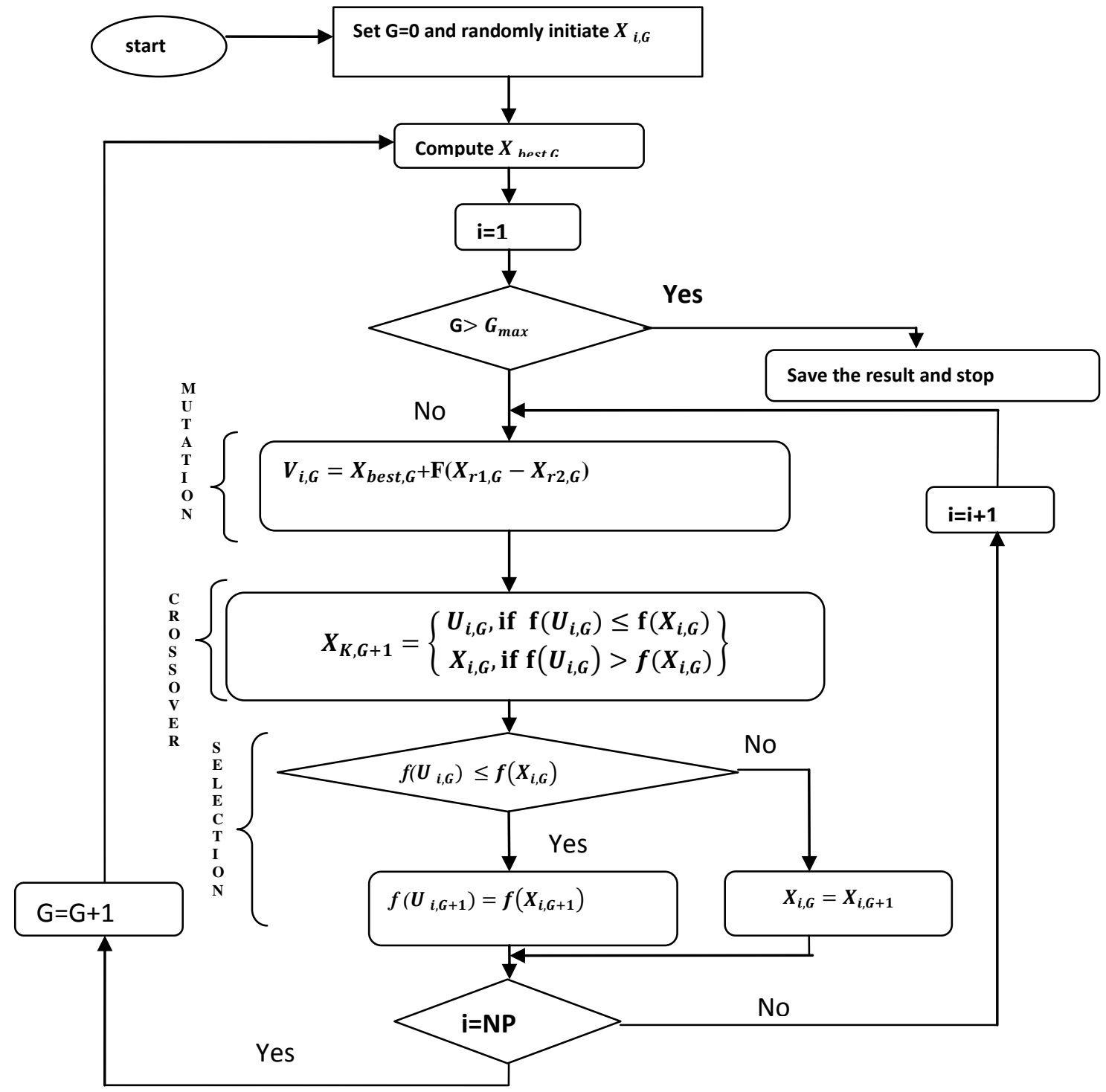

Fig4: DE Algorithm for optimization of SLL

\section{Resuits And Discussions}

In this paper, the Non-Uniform linear antenna array is synthesized using global optimizing techniques called PSO and DE algorithms. The Non-Uniform array is symmetric array of $2 \mathrm{~N}$ elements towards the center of the array. The array having progressive phase and uniform spacing between the elements is $0.5 \lambda$.The radiation pattern of linear antenna array with $\mathrm{N}=32$ elements at scan angles $0^{\circ}$ and $60^{\circ}$ deg are given for both PSO and DE. In this paper, the array factor is the objective function for optimizing the fitness /cost function called SLL. By using this evaluation techniques the best weights are obtained for linear antenna arrays to reduce the SLL. 

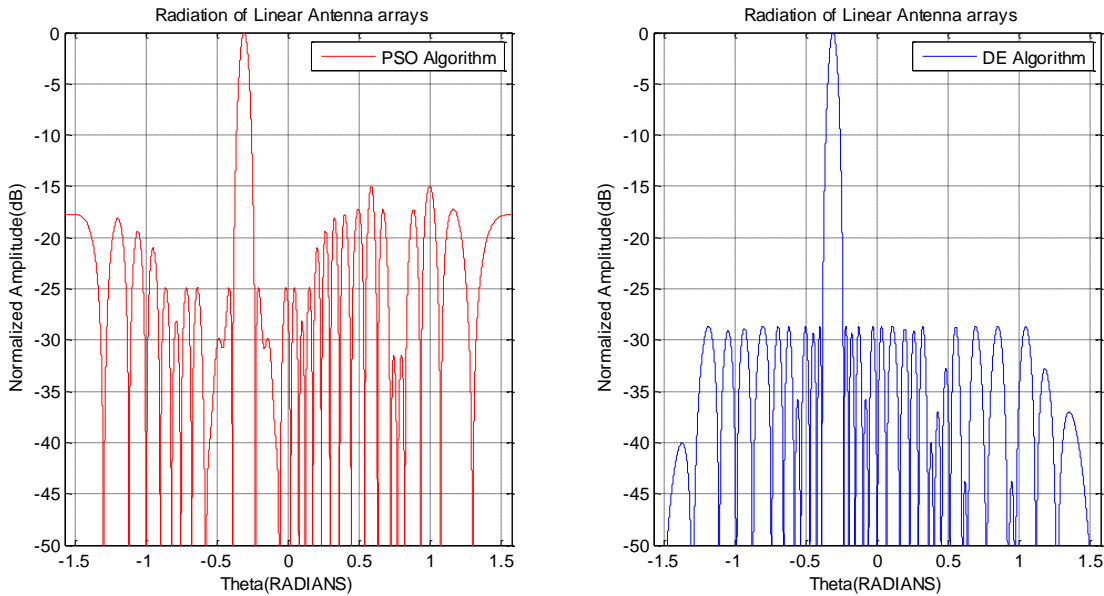

Fig5:Radiation Pattern of Linear antenna array of 20 elements using PSO and DE algorithms at $\theta=60^{\circ}(\mathrm{deg})$
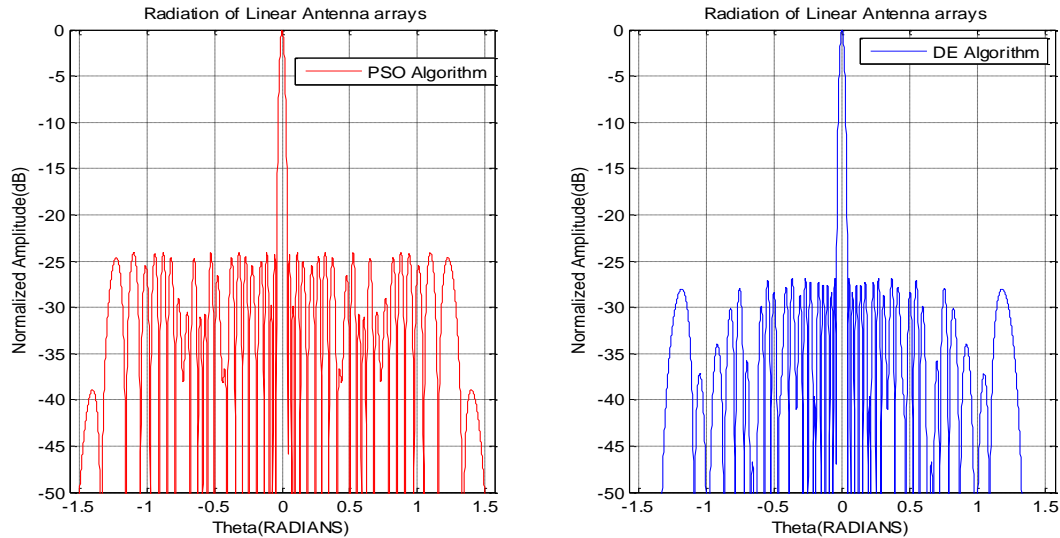

Fig6:Radiation Pattern of Linear antenna array of 32 elements using PSO and DE algorithms at $\theta=0^{\circ}(\mathrm{deg})$

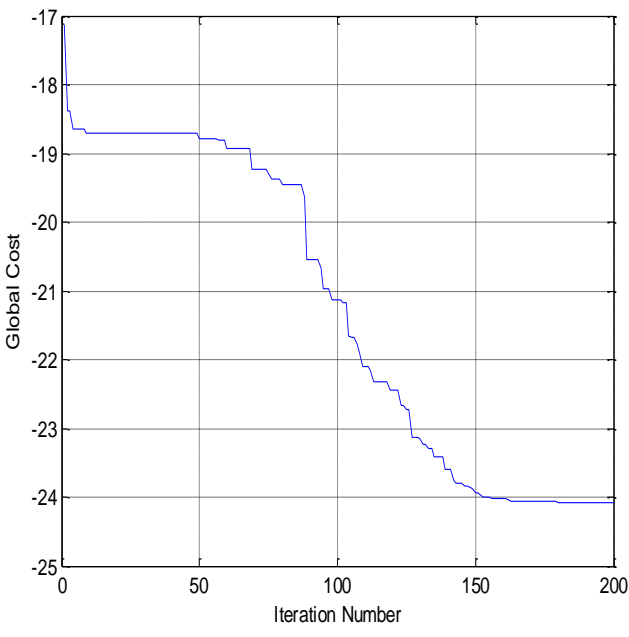

Fig 7: Cost function of PSO for

Linear antenna arrays

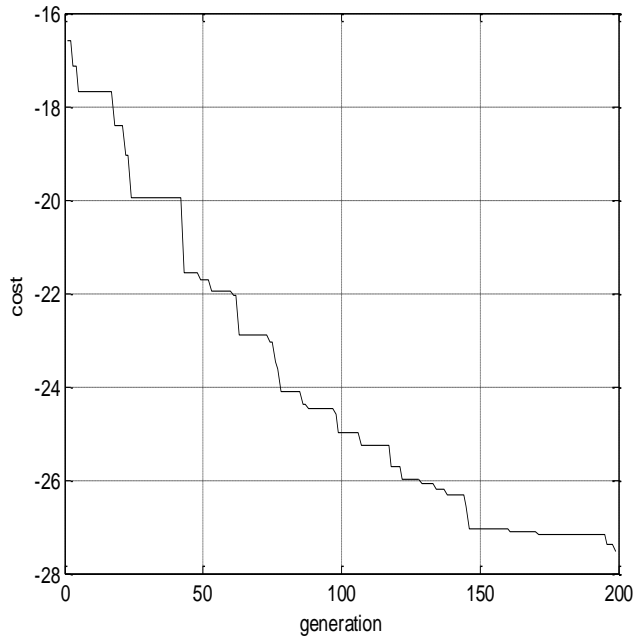

Fig 8: Cost function of DE Linear antenna arrays 


\begin{tabular}{|c|c|c|}
\hline S.No & $\begin{array}{l}\text { Optimized weights using } \\
\text { PSO }(\mathrm{N}=32)\end{array}$ & $\begin{array}{l}\text { Optimized weights using } \\
\text { DE }(\mathrm{N}=32)\end{array}$ \\
\hline 1 & 0.30852 & 0.2590 \\
\hline 2 & 0.12926 & 0.4895 \\
\hline 3 & 0.020026 & 0.3698 \\
\hline 4 & 0.64357 & 0.2998 \\
\hline 5 & 0.37447 & 0.3732 \\
\hline 6 & 0.20391 & 0.3317 \\
\hline 7 & 0.47834 & 0.2754 \\
\hline 8 & 0.32186 & 0.4176 \\
\hline 9 & 1.0000 & 0.4764 \\
\hline 10 & 0.38062 & 0.4683 \\
\hline 11 & 0.81723 & 0.5115 \\
\hline 12 & 0.57215 & 0.3315 \\
\hline 13 & 0.21467 & 0.7321 \\
\hline 14 & 0.37472 & 0.5820 \\
\hline 15 & 0.43875 & 0.3956 \\
\hline 16 & 0.81005 & 0.6280 \\
\hline 17 & 0.99999 & 0.8905 \\
\hline 18 & 1.0000 & 0.8068 \\
\hline 19 & 0.52906 & 0.8150 \\
\hline 20 & 1.0000 & 0.6646 \\
\hline 21 & 1.0000 & 0.6483 \\
\hline 21 & 1.0000 & 0.8901 \\
\hline 23 & 0.80843 & 0.7699 \\
\hline 24 & 1.0000 & 1.0000 \\
\hline 25 & 0.88378 & 0.7773 \\
\hline 26 & 1.0000 & 0.9159 \\
\hline 27 & 0.99991 & 0.8588 \\
\hline 28 & 1.0000 & 1.0000 \\
\hline 29 & 1.0000 & 0.9440 \\
\hline 30 & 1.0000 & 0.9719 \\
\hline 31 & 1.0000 & 0.9299 \\
\hline 32 & 1.00000 & 0.8618 \\
\hline
\end{tabular}

TABLE 1: Best weights for PSO and DE Algorithms

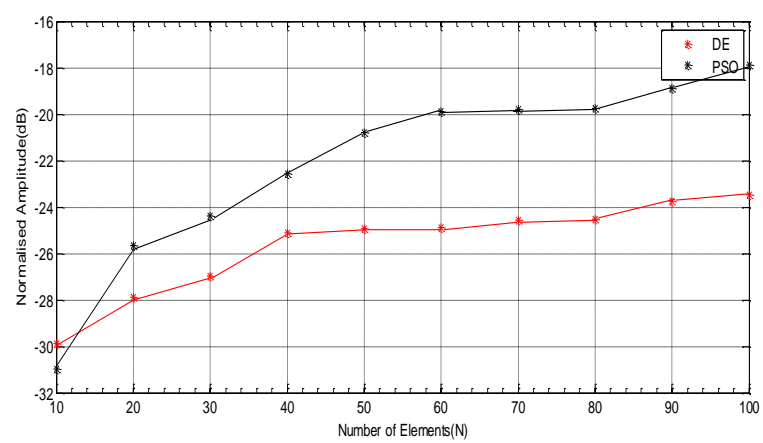

Fig 9: Maximum Sidelobe level with the number of elements.* DEA based non-uniform antenna array with uniform spacing $0.5 \lambda,{ }^{*}$ PSO based non-uniform antenna array with uniform spacing $0.5 \lambda$.

In $\mathrm{DE}$ Algorithm, the crossover $\mathrm{Cr}=0.5$, the mutation process having step size $\mathrm{F}=0.4$ was taken. The upper and lower bounds for each parameter are in the range of [0,1].Nearly 10 to 14 Strategies ,in which strategy 7 (DE/rand/1/bin) is used for better results for random number of generations are required. Number of population members is 16 . The dimensions of the problem $\mathrm{D}=\mathrm{N}=32$. In case of PSO, Number of population members is 16 , and $\mathrm{N}=32$. The constraint factors $\mathrm{C} 1=\mathrm{C} 2=2$ are taken. Fig 4: Represents the radiation pattern of Linear antenna array of 20 elements using PSO algorithm and DE algorithm at $\theta=60^{\circ}(\mathrm{deg})$, the SLL is $-24.98 \mathrm{~dB}$ and $-28.6 \mathrm{~dB}$ obtained respectively.Fig5: Represents the radiation pattern of Linear antenna array of 32 elements using PSO and DE algorithms at $\theta=0^{\circ}(\mathrm{deg})$, the SLL is $-24.09 \mathrm{~dB}$ and $-27.54 \mathrm{~dB}$ obtained respectively. Fig 6, Fig 7 represents the cost functions of PSO and DE algorithms of 200 iterations. Both the algorithm produced best results than normal linear antenna array having -13.6dB.Fig 9 represents Maximum Sidelobe 
level with the number of elements for both PSO and DE algorithms.From Fig 9, DE produces maximum SLL than PSO as number of elements increases. The simulation results are obtained using MATLAB software.

\section{Conclusion}

In this paper, the two efficient evolutionary techniques called Particle Swarm Optimization algorithm andDifferential evaluation algorithms were used for optimizing the non uniform linear antenna array in the direction of far field . Both PSO and DE optimized the SLL of non uniform linear antenna array with N=32 are $-24.09 \mathrm{~dB}$ and $27.5 \mathrm{~dB}$ respectively Without using any optimization techniques the SLL of linear antenna array is $-13.6 \mathrm{~dB}$.By simulating over 20-25 times PSO obtained SLL as $-24.09 \mathrm{~dB}$,but DE converges best result faster than PSO. The simulation result shows that DE has better optimum SLL than PSO algorithm. As the number of elements increases the DE gives best optimum values than PSO.

\section{References}

[1] Rahmat-Samii, Y., and Michielssen, E.: Electromagnetic Optimization by Genetic Algorithms. (New York: Wiley-Interscience, 1999)

[2] Robinson, J., and Rahmat-Samii, Y.: Particle swarm optimization in electromagnetics. IEEE Transactions on Antennas and Propagation, 2004, Vol.52, pp. 397-407.

[3] Storn, R. and K. Price, "Differential evolution-A simple and efficient heuristic for global optimization over continuous spaces," Journal of Global Optimization, Vol. 11, No. 4, 341-359, 1997.

[4] M. M. Khodier and C. G. Christodoulou, "Linear array geometry synthesis with minimum side lobe level and null control using particle swarm optimization," IEEE Transactions on Antennas and Propagation, Vol. 53, No. 8, August 2005.

[5] S. Yang, Y. B. Gan, and A. Qing, "Sideband suppression in time modulated linear arrays by the differential evolution algorithm," IEEEAntennas Wireless Propagat. Lett., vol. 1, pp. 173-175, 2002.

[6] D. Gies and Y. Rahmat-Samii, "Particle swarm optimization for reconfigurable phased-differentiated array design," Microw. Opt. Tech. Lett.,vol. 38, no. 3, pp. 168-175, Aug. 2003.

[7] Kurup, D., Himdi, M., and Rydberg, A.: Synthesis of uniform amplitude unequally spaced antenna arrays using the differential algorithm, IEEE Trans.Antennas Propagation, 2003, Vol. 51, pp. 2210-2217.

[8] F. Soltankarimi1, J. Nourinia and Ch. Ghobadi," Side Lobe Level Optimization in Phased Array Antennas Using Genetic Algorithm", ISSST A2004, Sydney, Australia, 30 Aug. - 2 Sep 2004.

[9] J. Kennedy, R. Eberhart, "Particle Swarm Optimization", IEEE Int. Conf. Neural Networks (Perth, Australia), vol. 4, 1995, pp.1942-1948.

[10] S. Das, P. N. Suganthan, "Differential Evolution: A survey of the Stateof- the-Art," IEEE Transactions on Evolutionary Computation, vol. 15, no. 1, pp. 4-31, 2011

[11] Y. Wang, Z. Cai, and Q. Zhang "Differential evolution with composite trial vector generation strategies and control parameters", IEEE Transactions on Evolutionary Computation, vol. 15, no. 1, pp. 55-66, 2011.

[12] J.Robinson,Y.Rahmat-Samii,"ParticleSwarm Optimization (PSO) in Electromagnetics", IEEE Trans. Antennas Propagation, vol.52,no.2,February 2004, pp. 397-407.

[13] P.J.Bevelacqua and C.A.Balanis,"Minimum sidelobe levels for linear arrays",IEEE Trans.Antennas Propagation., Vol.55,No.12,PP.3442-3449,Dec2007.

[14] K.Karuna Kumari, Dr.P.V.Sridevi Performance Evaluation of Circular Microstrip Patch Array with Difference Dielectric Substrate Materials "IJECET volume 4,issue 1 January-Feb-2013,pp236-249

[15] K.Karuna Kumari, Dr.P.V.Sridevi "Side lobe level optimization of circular Microstrip array antenna using Genetic Algorithm" IJECIERD Volume 4,issue 1 January-Feb-2014pp 9-18

[16] F.T. Tsen`g and D.K. Cheng, "Optimum scannable planar arrays with an invariant sidelobe level," $P$. IEEE, vol. 56, no. 11, pp. 1771-1778, Nov.1968

[17] S. Yang, Y. B. Gan, and A. Qing, "Sideband suppression in time modulated linear arrays by the differential evolution algorithm," IEEEAntennas Wireless Propagat. Lett., vol. 1, pp. 173-175, 2002.

[18] Nanbo Jin and Yahya Rahmat ,'Hybrid Real-Binary Particle Swarm Optimization in Engineering Electromagnetics",IEEE Trans.Antennas and Propagation,Vol.58.No-12,Dec-2010.

\section{BOOKS}

[19] Balanis, C.: Antenna Theory-Analysis and Design. (2nd Edition New York:Wiley, 1997)

[20] James Blondin, Particle Swarm Optimization: A Tutorial September4, 2009. 